\title{
SSTR2A expression in medullary thyroid carcinoma is correlated with longer survival
}

\author{
Lisa H. de Vries ${ }^{1} \cdot$ Lutske Lodewijk $^{1} \cdot$ Stefan M. Willems ${ }^{2} \cdot$ Koen M. A. Dreijerink ${ }^{3}$ - Bart de Keizer ${ }^{4}$. \\ Paul J. van Diest ${ }^{2} \cdot$ Abbey Schepers $^{5} \cdot$ Han J. Bonenkamp ${ }^{6}$. Ilse A. C. H. van Engen-van Grunsven ${ }^{7}$. Schelto Kruijff ${ }^{8}$. \\ Bettien M. van Hemel ${ }^{9}$. Thera P. Links ${ }^{10}$. Els J. M. Nieveen van Dijkum ${ }^{11} \cdot$ Susanne van Eeden ${ }^{12} \cdot$ Gerlof D. Valk $^{3}$. \\ Inne H. M. Borel Rinkes ${ }^{1} \cdot$ Menno R. Vriens ${ }^{1}$
}

Received: 1 May 2018 / Accepted: 27 July 2018 / Published online: 20 August 2018

(c) The Author(s) 2018

\begin{abstract}
Purpose Medullary thyroid carcinoma (MTC) derives from the parafollicular C-cells of the thyroid gland. Somatostatin receptors (SSTRs) are expressed in various neuroendocrine tumours including MTC. The aim of this study was to evaluate SSTR2A as a prognostic factor for MTC, to study distribution of SSTR2A expression within tumours and to compare expression of SSTR2A between primary tumours and corresponding lymph node metastases.

Methods Patients who underwent surgery between 1988 and 2014 for MTC from five tertiary referral centres in The Netherlands were included. In total, primary tumours of 114 patients and lymph node metastases of 34 patients were analysed for expression of SSTR2A using a tissue microarray, and correlated with clinicopathological variables and survival. Results The mean age of patients was 45.5 years (SD 16.2), 55 patients were male (49.5\%). Primary tumours of 58 patients (50.9\%) showed SSTR2A expression. In multivariate Cox-regression analysis, SSTR2A positivity correlated independently with better overall survival (OS) (HR 0.3; 95\% CI 0.1-1.0). In stage IV MTC patients, 10-year survival rates for SSTR2Anegative and positive patients were $43 \%$ and $96 \%$, respectively. In $53.9 \%$ of patients with lymph node metastases, expression in primary tumour and lymph node metastases differed.

Conclusion SSTR2A expression is correlated with longer OS in MTC, especially for stage IV patients, suggesting that SSTR2A expression might be a useful prognostic factor in MTC. The SSTR2A status of the primary MTC does not predict expression in lymph node metastases.
\end{abstract}

Keywords Medullary thyroid carcinoma $\cdot$ Immunohistochemistry $\cdot$ Somatostatin receptor $2 \mathrm{~A} \cdot$ Tissue microarray $\cdot$ Oncology

Electronic supplementary material The online version of this article (https://doi.org/10.1007/s12020-018-1706-1) contains supplementary material, which is available to authorised users.

Menno R. Vriens

mvriens@umcutrecht.nl

1 Department of Surgery, University Medical Centre Utrecht, Heidelberglaan 100, 3584CX Utrecht, The Netherlands

2 Department of Pathology, University Medical Centre Utrecht, Heidelberglaan 100, 3584CX Utrecht, The Netherlands

3 Department of Endocrine Oncology, University Medical Centre Utrecht, Heidelberglaan 100, 3584CX Utrecht, The Netherlands

4 Department of Radiology, University Medical Centre Utrecht, Heidelberglaan 100, 3584CX Utrecht, The Netherlands

5 Department of Surgery, Leiden University Medical Centre, Albinusdreef 2, 2333ZA Leiden, The Netherlands

6 Department of Surgery, Radboud University Medical Centre,
Geert Grooteplein 8, 6525GA Nijmegen, The Netherlands

7 Department of Pathology, Radboud University Medical Centre, Geert Grooteplein 8, 6525GA Nijmegen, The Netherlands

8 Department of Surgery, University Medical Centre Groningen, Hanzeplein 1, 9700RB Groningen, The Netherlands

9 Department of Pathology, University Medical Centre Groningen, Hanzeplein 1, 9700RB Groningen, The Netherlands

10 Department of Internal Medicine, University Medical Centre Groningen, Hanzeplein 1, 9700RB Groningen, The Netherlands

11 Department of Surgery, Academic Medical Centre Amsterdam, Meibergdreef 9, 1105AZ Amsterdam, The Netherlands

12 Department of Pathology, Academic Medical Centre Amsterdam, Meibergdreef 9, 1105AZ Amsterdam, The Netherlands 


\section{Introduction}

Medullary thyroid carcinoma (MTC) is derived from the parafollicular clear cells (C-cells) of the thyroid gland [1]. Although MTC accounts for only $4 \%$ of all thyroid carcinomas, it is accountable for about $13 \%$ of deaths resulting from thyroid cancer [2]. Besides a sporadic form that occurs in the majority of cases, MTC develops in the remaining $25-30 \%$ of patients as part of the hereditary tumour syndrome Multiple Endocrine Neoplasia type 2 (MEN2) [1]. Patients who are treated with curative intent should always have a total thyroidectomy and dissection of the central lymph node compartment. Adjuvant therapy in general is of limited value [3-5]. Unfortunately, in more than $50 \%$ of patients, calcitonin levels remain elevated after supposed curative resection, indicating that subclinical active tumour tissue is still present $[3,6]$.

Early diagnosis of MTC is very important to improve prognosis. When diagnosed in an early phase (stage I and II), 10-year survival rates are favourable at $100 \%$ and $93 \%$, respectively. Unfortunately, approximately $45 \%$ of patients present with metastases (stage III and IV), associated with 10 -year survival rates of $71 \%$ and $21 \%$, respectively [3]. Prognosis is difficult to predict in stage III or IV MTC, whereas this is essential for personalised follow-up and adjuvant therapy strategies for patients that will rapidly progress [7].

The C-cells of the thyroid gland have the ability to express somatostatin receptors (SSTRs) like many neuroendocrine tumours. There are six different subtypes: SSTR1, SSTR2A, SSTR2B, SSTR3, SSTR4 and SSTR5 [8]. These receptors have a broad spectrum of biological actions including inhibition of proliferation, cell-survival and angiogenesis [8-10]. In this study, we focused on SSTR2A since this particular receptor is used as a target for nuclear imaging as well as for medical therapy. Expression of SSTR2 enables the use of somatostatin analogues (SSTA) for therapeutic purposes, which has a positive effect on the symptoms accompanying MTC like diarrhoea, weight loss and malaise [11-13]. Unfortunately SSTA therapy does not reduce tumour mass, nor improve survival rates [12]. Peptide receptor radionuclide therapy (PRRT) with radioactively labelled SSTA shows promising results in other endocrine tumours, with much higher response rates than SSTA therapy. In a recent randomised phase 3 trial in patients with progressive midgut neuroendocrine tumours, treatment with ${ }^{177} \mathrm{Lu}$-Dotatate resulted in a risk of progression or death that was $79 \%$ lower than the risk associated with a high-dose of the SSTA octreotide [14]. Limited information is available on the effect of PRRT with SSTA in MTC. Only a few, small sample sized studies have been conducted, but with promising results. Disease progression, stable disease and (partial) response was seen in
$14-65 \%, 0-61 \%$ and $18-67 \%$ of patients, respectively. Toxicity was low and quality of life improved using PRRT [15-18]. To determine whether a patient is eligible for treatment with PRRT, the receptor subtype and the amount of expression in this specific tumour needs to be established $[15,18]$. This can be done by imaging using radioactive SSTA or by immunohistochemistry on tumour tissue [11].

Currently, imaging modalities still have limitations in identifying metastases, resulting in a low specificity and sensitivity [3]. SSTR expression enables the use of SSTA scans for follow-up in patients with persistent MTC for localisation of metastases, especially in the neck lymph nodes [12]. At this moment, stability of SSTR expression between primary tumours and lymph node metastases is not yet investigated in MTC. For the utility of SSTA scans, such information is necessary.

Besides being useful as therapeutic target, SSTR expression might be relevant as a prognostic factor. Herac et al. [8]. studied the correlation between SSTR2A and SSTR5 expression and several clinicopathological features. They demonstrated a significant correlation between SSTR2A expression and the presence of lymph node metastases, which is an indicator of poor prognosis, but no survival analysis was presented $[3,8]$. The aim of this study was to evaluate SSTR2A as a prognostic factor in a larger cohort of MTC patients with long-term follow-up. Furthermore, we studied distribution of SSTR2A within tumours and compared expression of SSTR2A between primary tumours and corresponding lymph node metastases.

\section{Materials and methods}

\section{Patients}

Patients who underwent surgery between 1988 and 2014 for MTC were identified from the pathology databases of Leiden University Medical Centre (LUMC), Amsterdam Medical Centre (AMC), Radboud University Medical Centre (RadboudUMC), University Medical Centre Groningen (UMCG) and University Medical Centre Utrecht (UMCU), The Netherlands (all tertiary referral centres). Formalin fixed paraffin embedded (FFPE) tissues were collected from the pathology archives. In total, 114 patients were identified from whom primary tumour tissue was available for inclusion in the tissue microarray (TMA). From two of the five hospitals (LUMC and UMCU), tissue of lymph node metastases was available as well, enabling analysis of lymph node tissue of 34 patients.

Clinical and pathological patient information was retrieved from patient files in all five centres. All MEN2 diagnoses were confirmed by germline mutation analysis of the RET gene. Sporadic patients were either patients with 

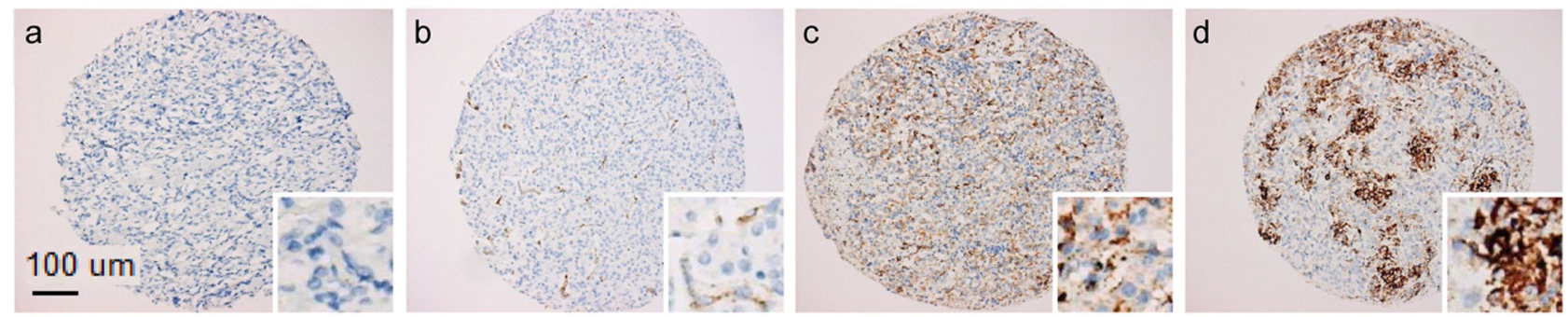

Fig. 1 Representative examples of immunohistochemical staining for SSTR2A in TMA of MTC. a Absent SSTR2A staining. b SSTR2A staining with intensity 1 in $10 \%$ of cells. c SSTR2A staining with intensity 2 in $100 \%$ of cells. d SSTR2A staining with intensity 3 in $60 \%$ of cells

negative germline mutation analysis or with a negative family history. Microscopic positive resection margins were considered as part of the T-stage and not included as a separate variable. Disease status was based on postoperative (dichotomous) calcitonin and CEA serum values. No exact values or doubling times were considered since we included patients from five centres over almost three decades for which different assays were applied at the time. An elevation in CEA or calcitonin was interpreted as persistent disease, CEA or calcitonin within normal range was interpreted as cured. Only postoperative CEA and calcitonin values that were measured $>6$ months after surgery were taken into account. Whole slides were scored for necrosis, angioinvasion and desmoplasia. Necrosis and angioinvasion were scored as absent or present and desmoplasia as negative, some, moderate or severe. These scorings were performed on the same FFPE blocks that were used for the construction of the TMA.

This study was performed according to national guidelines with respect to the use of leftover tissue and approval for this study, including the use of patient data, was obtained from the Institutional Review Board of the UMCU [7, 19].

\section{Construction of tissue microarray}

The TMA was constructed using an automated machine (TMA grand master, 3D Histec, Budapest, Hungary). Three cores of $0.6 \mathrm{~mm}$ were punched from each FFPE block of primary tumour and lymph node metastases if available. To assure that cores were punched from tumour areas, cell rich areas were marked on H\&E slides by a pathologist (P.v.D. and L.L.), scanned, and marks were manually circled with the TMA software (3D Histech).

\section{Immunohistochemistry}

The TMA blocks were cut at $4 \mu \mathrm{m}$ and mounted on coated slides. Staining for SSTR2A was carried out with an automatic immunostainer (Bench Mark ULTRA Automated IHC slide staining system, Ventana Medical Systems, Inc.,
USA) following protocol: after baking the slides at $75{ }^{\circ} \mathrm{C}$ and incubating for eight minutes, the slides were deparaffinised on $72{ }^{\circ} \mathrm{C}$. Antigen retrieval was performed using the $\mathrm{CC} 1$ standard pre-treatment. The primary SSTR2A antibody (1:20; code SS-8000-RM(A); rabbit IgG; biotrend) was incubated for $32 \mathrm{~min}$ at $37^{\circ} \mathrm{C}$. Slides were counterstained with haematoxylin and coverslipped.

\section{Scoring of immunohistochemistry}

All cores in the SSTR2A TMA slides were scored by an experienced pathologist (S.M.W.) and an experienced researcher (L.L.) for staining intensity as absent (0), weak (1), moderate (2) or strong (3) and percentage of positive tumour cells. On the basis of Volante et al. [20], we considered only membranous staining positive and discarded cytoplasmic staining. Representative scores of all immunostainings are shown in Fig. 1. Data on hypoxia inducible factor-1 alpha (HIF-1 $\alpha$ ), vascular endothelial growth factor (VEGF), glucose transporter 1 (Glut-1), carbonic anhydrase IX (CAIX) and microvessel density (MVD) was available from a previous study [7].

\section{Statistical analysis}

Categorical data were summarised with frequencies and percentages, and continuous data were summarised with medians and ranges. To increase the power of the statistical analysis categorical data were recoded into dichotomous variables. Grade of desmoplasia was recoded into none-some and moderate-severe. Stage was recoded into stage I-III and stage IV. Hereditability was recoded in either sporadic disease or MEN2 syndrome [7]. SSTR2A scorings were transformed into a dichotomous variable, being positive when the average intensity of the three cores was $\geq 1$, independently of the previously scored percentage of staining.

Overall survival (OS) was defined as the time to death from any cause. Progression-free survival (PFS) was defined as the time to development of distant metastases or death.

Univariate Kaplan-Meier survival analysis was performed and Kaplan-Meier survival curves were plotted. 
The log-rank test was used to calculate significance. Multivariate Cox-regression analysis was performed and hazard ratios (HR) of clinicopathological characteristics on OS were calculated. All reported $p$-values were two sided. Analysis was performed using SPSS version 24.0 software (SPSS, Inc., Chicago, IL, USA).

\section{Results}

\section{Clinicopathological variables}

The 114 patients included in this study were between eight and 82 years of age (mean age 45.5, SD 16.2). Fifty-five patients were male $(49.5 \%)$ and $56(50.5 \%)$ were female (in three cases gender was unknown). Sixty patients (57.7\%) had sporadic MTC, 40 patients (38.5\%) had MEN2a and four patients had MEN2b (3.8\%) (in ten patients the mutation status was unknown). Median tumour size ranged from 4 to $70 \mathrm{~mm}$ (mean $26.1 \mathrm{~mm}$ ). At the time of first surgery 68 patients $(61.8 \%)$ presented with lymph node metastases. Fifteen patients (14.4\%) presented with stage I, $25(24.0 \%)$ with stage II, $16(15.4 \%)$ with stage III and 48 $(46.2 \%)$ with stage IV (in ten patients tumour stage was unknown). Mean follow-up time was 69.5 months (range 0-318 months).

\section{SSTR2A expression in primary tumours}

Sixty-five patients (57.0\%) expressed SSTR2A in their primary tumour, including weak expression (average intensity $\geq 1)$ in 35 patients $(30.7 \%)$, moderate expression (average intensity $\geq 2$ ) in 14 patients $(12.3 \%)$ and strong expression (average intensity 3 ) in nine patients (7.9\%). In the remaining seven patients $(6.1 \%)$, so little expression (average $<1$ ) was seen that these patients were classified as negative. In 49 patients (43.0\%) there was no SSTR2A expression present in the primary tumour. Figure 2 shows the intensity of all scored cores in each patient individually and demonstrates the distribution of different intensities throughout the entire group of patients.

As to primary tumour distribution of SSTR2A expression, ten patients had only one core and were discarded. Of the remaining 104 patients, 72 (69.3\%) showed homogeneous expression and 32 (30.8\%) showed heterogeneous expression.

\section{SSTR2A expression in primary tumours vs. lymph node metastases}

The average SSTR2A intensity was 0.9 (SD 1.0) of all primary tumours $(n=114)$ and 0.7 (SD 1.1), 0.8 (SD 1.0) and 0.6 (SD 0.7) for lymph node metastases at initial presentation and subsequent surgeries (not significant).

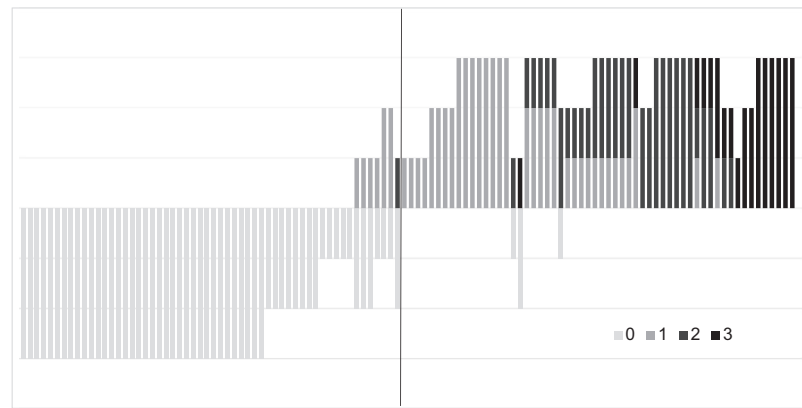

Fig. 2 Intensity of all scored cores in each patient individually arranged from low average intensity to high average intensity. Each bar represents one patient. The length of the bar correlates with the amount of cores scored per patient. The colours of the bars represent the intensity of the staining. The patients on the left of the blue stripe are SSTR2A negative, the patients on the right of the blue stripe are SSTR2A positive

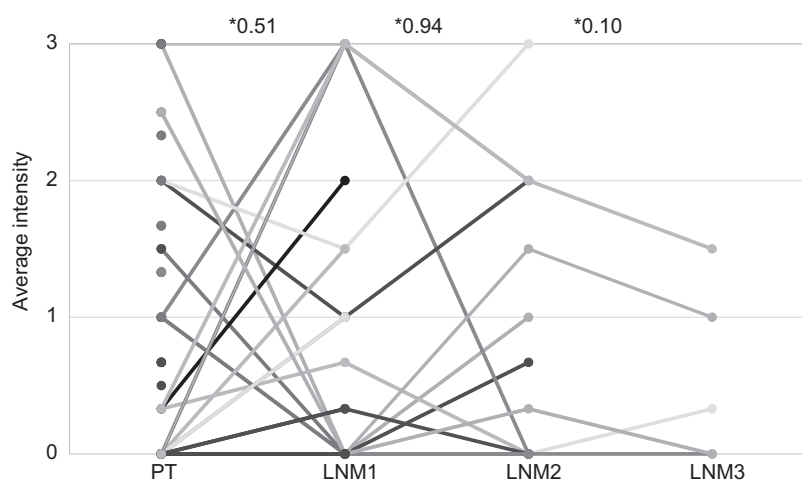

Fig. 3 Average intensity of SSTR2A expression in primary tumour and their subsequent lymph node metastases. The vertical axis shows the average intensity of the staining. PT primary tumour, LNM lymph node metastasis. $*=p$-value between average intensities calculated by Wilcoxon Signed Ranks Test

Figure 3 shows the average expression within the various tissues for each individual patient. Five patients (14.7\%) showed SSTR2A expression in both primary tumour and all lymph node metastases, but with differences in intensity levels between the primary tumour and lymph node metastases in all five. Eleven patients (32.4\%) showed no SSTR2A expression at all in either tissue. Ten patients (29.4\%) did not express any SSTR2A in the primary tumour but did show SSTR2A expression in $\geq 1$ of the lymph node metastases. Eight patients $(23.5 \%)$ showed SSTR2A expression in the primary tumour but no expression in $\geq 1$ of the lymph nodes metastases.

\section{Association between primary tumour SSTR2A expression and clinicopathological variables}

Expression of SSTR2A in comparison with clinicopathological parameters is outlined in Table 1. Heritability (MEN2) and HIF-1 $\alpha$ were the only characteristics 
Table 1 Clinicopathological characteristics of MTC patients stratified by SSTR2A intensity

\begin{tabular}{|c|c|c|c|c|c|}
\hline \multirow[b]{3}{*}{ Mean age in years (SD) } & \multirow{2}{*}{\multicolumn{2}{|c|}{$\begin{array}{l}\begin{array}{l}\text { SSTR2A } \\
\text { negative }\end{array} \\
N=56\end{array}$}} & \multirow{2}{*}{\multicolumn{2}{|c|}{$\begin{array}{l}\begin{array}{l}\text { SSTR2A } \\
\text { positive }\end{array} \\
N=58\end{array}$}} & \multirow{3}{*}{$\frac{p \text {-value }}{0.90}$} \\
\hline & & & & & \\
\hline & 45.3 & $(16.7)$ & 52 & $(15.8)$ & \\
\hline Gender & & & & & 1.00 \\
\hline Male $(\%)$ & 28 & $(50.9)$ & 27 & $(49.1)$ & \\
\hline Female $(\%)$ & 28 & $(50.0)$ & 28 & $(50)$ & \\
\hline Heritability & & & & & 0.01 \\
\hline Sporadic $(\%)$ & 38 & (63.3) & 22 & $(36.7)$ & \\
\hline MEN2a/b (\%) & 15 & $(34.1)$ & 29 & $(65.9)$ & \\
\hline Stage & & & & & 0.56 \\
\hline I-III (\%) & 28 & $(51.9)$ & 26 & $(48.1)$ & \\
\hline IV (\%) & 22 & $(45.8)$ & 26 & $(54.2)$ & \\
\hline Mean size in mm (SD) & 26.8 & $(14.4)$ & 25.4 & $(15.7)$ & 0.64 \\
\hline$<20 \mathrm{~mm}(\%)$ & 17 & $(45.9)$ & 20 & $(54.1)$ & 0.67 \\
\hline$\geq 20 \mathrm{~mm}(\%)$ & 32 & $(52.5)$ & 29 & $(47.5)$ & \\
\hline Lymph node metastasis & & & & & 0.08 \\
\hline No $(\%)$ & 26 & $(61.9)$ & 16 & $(38.1)$ & \\
\hline Yes $(\%)$ & 29 & $(42.6)$ & 39 & $(57.4)$ & \\
\hline Overall survival & & & & & 0.06 \\
\hline Did not die $(\%)$ & 38 & $(44.2)$ & 48 & $(55.8)$ & \\
\hline Died $(\%)$ & 9 & $(75.0)$ & 3 & $(25.0)$ & \\
\hline Progression-free survival (\%) & & & & & 0.41 \\
\hline No progression/death & 40 & $(51.3)$ & 38 & $(48.7)$ & \\
\hline Progression/death & 12 & $(46.2)$ & 14 & $(53.8)$ & \\
\hline Disease status & & & & & 0.15 \\
\hline Normal CEA $(\%)$ & 14 & $(37.8)$ & 23 & $(62.2)$ & \\
\hline Elevated CEA (\%) & 33 & $(54.1)$ & 28 & $(45.9)$ & \\
\hline Necrosis & & & & & 1.00 \\
\hline Absent $(\%)$ & 43 & $(47.8)$ & 47 & $(52.2)$ & \\
\hline Present $(\%)$ & 4 & $(44.4)$ & 5 & $(55.6)$ & \\
\hline Angioinvasion & & & & & 0.32 \\
\hline Absent $(\%)$ & 44 & $(49.4)$ & 45 & $(50.6)$ & \\
\hline Present $(\%)$ & 3 & $(30.0)$ & 7 & $(70.0)$ & \\
\hline Desmoplasia & & & & & 0.23 \\
\hline None-some (\%) & 27 & $(54.0)$ & 23 & $(46.0)$ & \\
\hline Moderate-severe $(\%)$ & 20 & $(40.8)$ & 29 & $(59.2)$ & \\
\hline HIF- $1 \alpha$ & & & & & 0.00 \\
\hline Negative $(\%)$ & 30 & $(68.2)$ & 14 & $(31.8)$ & \\
\hline Positive $(\%)$ & 20 & (34.5) & 38 & $(65.5)$ & \\
\hline CAIX & & & & & 0.43 \\
\hline Negative (\%) & 28 & $(52.8)$ & 25 & $(47.2)$ & \\
\hline Positive (\%) & 21 & $(43.8)$ & 27 & $(56.3)$ & \\
\hline Glut-1 & & & & & 0.21 \\
\hline Negative (\%) & 49 & $(51.0)$ & 47 & (49.0) & \\
\hline Positive (\%) & 1 & $(16.7)$ & 5 & $(83.3)$ & \\
\hline MVD (SD) & 14.5 & $(9.5)$ & 13.9 & (6.1) & 0.72 \\
\hline
\end{tabular}

Table 1 (continued)

\begin{tabular}{|c|c|c|c|c|c|}
\hline \multirow[b]{3}{*}{$<14.3$ vessels/core $(\%)$} & \multirow{2}{*}{\multicolumn{2}{|c|}{$\begin{array}{l}\text { SSTR2A } \\
\text { negative }\end{array}$}} & \multirow{2}{*}{\multicolumn{2}{|c|}{$\begin{array}{l}\begin{array}{l}\text { SSTR2A } \\
\text { positive }\end{array} \\
N=58\end{array}$}} & \multirow[t]{2}{*}{$p$-value } \\
\hline & & & & & \\
\hline & 30 & (53.6) & 26 & $(46.4)$ & 0.32 \\
\hline$\geq 14.3$ vessels/core $(\%)$ & 19 & $(42.2)$ & 26 & $(57.8)$ & \\
\hline VEGF & & & & & 0.84 \\
\hline Negative (\%) & 17 & $(45.9)$ & 20 & $(54.1)$ & \\
\hline Positive (\%) & 30 & $(49.2)$ & 31 & $(50.8)$ & \\
\hline
\end{tabular}

that were significantly different between SSTR2A-negative and positive groups with $34.1 \%$ vs. $65.9 \%$, and $34.5 \%$ vs. $65.5 \%$, respectively.

\section{Prognostic value}

As outlined in Table 2, for OS a significant prognostic association was found for SSTR2A expression, heritability, stage, lymph node metastases, disease status, necrosis and VEGF. Thirty-eight patients $(80.9 \%)$ vs. 48 patients (94.1\%) survived in the SSTR2A-negative and SSTR2Apositive groups, respectively, $(p=0.035)$. In the Kaplan-Meier curves shown in Fig. 4 the prognostic effect of SSTR2A positivity for all patients is shown, for patients with stage I-III and for patients with stage IV. Within the group of patients having stage IV MTC, 10-year survival rates decrease significantly in SSTR2A-negative patients; from $96 \%$ for SSTR2A-positive patients to $43 \%$ for SSTR2A-negative patients. In the small subpopulation of patients with lymph node metastases, 10-year survival rates decrease, although not significantly, when SSTR2A is not expressed in $\geq 1$ of the lymph nodes; from $64 \%$ for patients with $\geq 1$ positive lymph node to $49 \%$ for patients with negative lymph nodes as shown in Supplementary Fig. 1. For PFS a significant association was found for heritability, stage, lymph node metastases, disease status, necrosis, HIF$1 \alpha$ and Glut-1, but not for SSTR2A expression.

Twelve patients died, which restricted the number of variables we could use in multivariate Cox-regression analysis. We chose to include SSTR2A and stage, because disease stage is currently the strongest prognostic variable available. The variable heritability was left out of multivariate Cox-regression analysis since in our study almost all patients with hereditary tumours had stage I or II MTC. This can be explained by the fact that the majority of these patients had prophylactic thyroidectomies. Lymph node metastasis were not included since this variable is also taken into account when tumour stage is analysed. Disease status was left out because this is predominantly based on postoperative CEA/calcitonin measurements, which only have meaning when evaluated over time, but not as a single 
Table 2 Univariate survival analysis on progression-free (PFS) and overall survival (OS) for MTC patients

\begin{tabular}{|c|c|c|c|c|c|c|c|c|}
\hline \multirow{3}{*}{ SSTR2A } & \multicolumn{4}{|c|}{ Progression-free survival } & \multicolumn{4}{|c|}{ Overall survival } \\
\hline & $N$ & $\operatorname{PF}(N)$ & $\mathrm{PF}(\%)$ & $p$-value & $N$ & OS $(N)$ & OS $(\%)$ & $p$-value \\
\hline & & & & 0.71 & & & & 0.04 \\
\hline Negative & 52 & 40 & 76.9 & & 47 & 38 & 80.9 & \\
\hline Positive & 52 & 38 & 73.1 & & 51 & 48 & 94.1 & \\
\hline Gender & & & & 0.15 & & & & 0.48 \\
\hline Male & 51 & 35 & 68.6 & & 48 & 41 & 85.4 & \\
\hline Female & 53 & 43 & 81.1 & & 50 & 45 & 90.0 & \\
\hline Heritability & & & & 0.01 & & & & 0.00 \\
\hline Sporadic & 56 & 39 & 69.9 & & 52 & 42 & 80.8 & \\
\hline MEN2a/b & 44 & 37 & 84.1 & & 42 & 41 & 97.6 & \\
\hline Stage & & & & 0.00 & & & & 0.02 \\
\hline I-III & 53 & 51 & 96.2 & & 50 & 48 & 96.0 & \\
\hline IV & 47 & 23 & 48.9 & & 45 & 35 & 77.8 & \\
\hline Size in $\mathrm{mm}$ & & & & 0.35 & & & & 0.87 \\
\hline$<20 \mathrm{~mm}$ & 36 & 31 & 86.1 & & 36 & 33 & 91.7 & \\
\hline$\geq 20 \mathrm{~mm}$ & 60 & 18 & 70.0 & & 56 & 49 & 87.5 & \\
\hline Lymph node metastasis & & & & 0.00 & & & & 0.03 \\
\hline No & 40 & 39 & 97.5 & & 37 & 36 & 97.3 & \\
\hline Yes & 64 & 39 & 60.9 & & 61 & 50 & 82.0 & \\
\hline Disease status & & & & 0.00 & & & & 0.01 \\
\hline Normal CEA/calcitonin & 37 & 35 & 94.6 & & 37 & 37 & 100.0 & \\
\hline Elevated CEA/calcitonin & 60 & 36 & 60.0 & & 57 & 45 & 78.9 & \\
\hline Necrosis & & & & 0.00 & & & & 0.00 \\
\hline Absent & 88 & 68 & 77.3 & & 85 & 76 & 89.4 & \\
\hline Present & 9 & 3 & 33.3 & & 7 & 4 & 57.1 & \\
\hline Angioinvasion & & & & 0.11 & & & & 0.85 \\
\hline Absent & 87 & 66 & 75.9 & & 83 & 72 & 86.7 & \\
\hline Present & 10 & 5 & 50.0 & & 9 & 8 & 88.9 & \\
\hline Desmoplasia & & & & 0.33 & & & & 0.34 \\
\hline None-some & 50 & 40 & 80.0 & & 48 & 44 & 91.7 & \\
\hline Moderate-severe & 47 & 31 & 66.0 & & 44 & 36 & 81.8 & \\
\hline HIF- $1 \alpha$ & & & & 0.00 & & & & 0.06 \\
\hline Negative & 42 & 39 & 90.7 & & 41 & 39 & 95.1 & \\
\hline Positive & 57 & 22 & 61.4 & & 54 & 44 & 81.5 & \\
\hline CAIX & & & & 0.40 & & & & 0.25 \\
\hline Negative & 53 & 43 & 81.1 & & 52 & 48 & 92.3 & \\
\hline Positive & 46 & 31 & 67.4 & & 42 & 34 & 81.0 & \\
\hline Glut-1 & & & & 0.01 & & & & 0.52 \\
\hline Negative & 94 & 71 & 75.5 & & 90 & 78 & 86.7 & \\
\hline Positive & 6 & 3 & 50.0 & & 5 & 5 & 100.0 & \\
\hline MVD & & & & 0.31 & & & & 0.51 \\
\hline$<14.3$ vessels/core & 55 & 37 & 67.3 & & 51 & 43 & 84.3 & \\
\hline$\geq 14.3$ vessels/core & 44 & 36 & 81.8 & & 43 & 39 & 90.7 & \\
\hline VEGF & & & & 0.65 & & & & 0.03 \\
\hline Negative & 37 & 26 & 70.3 & & 34 & 32 & 94.1 & \\
\hline Positive & 59 & 46 & 78.0 & & 57 & 47 & 82.5 & \\
\hline
\end{tabular}



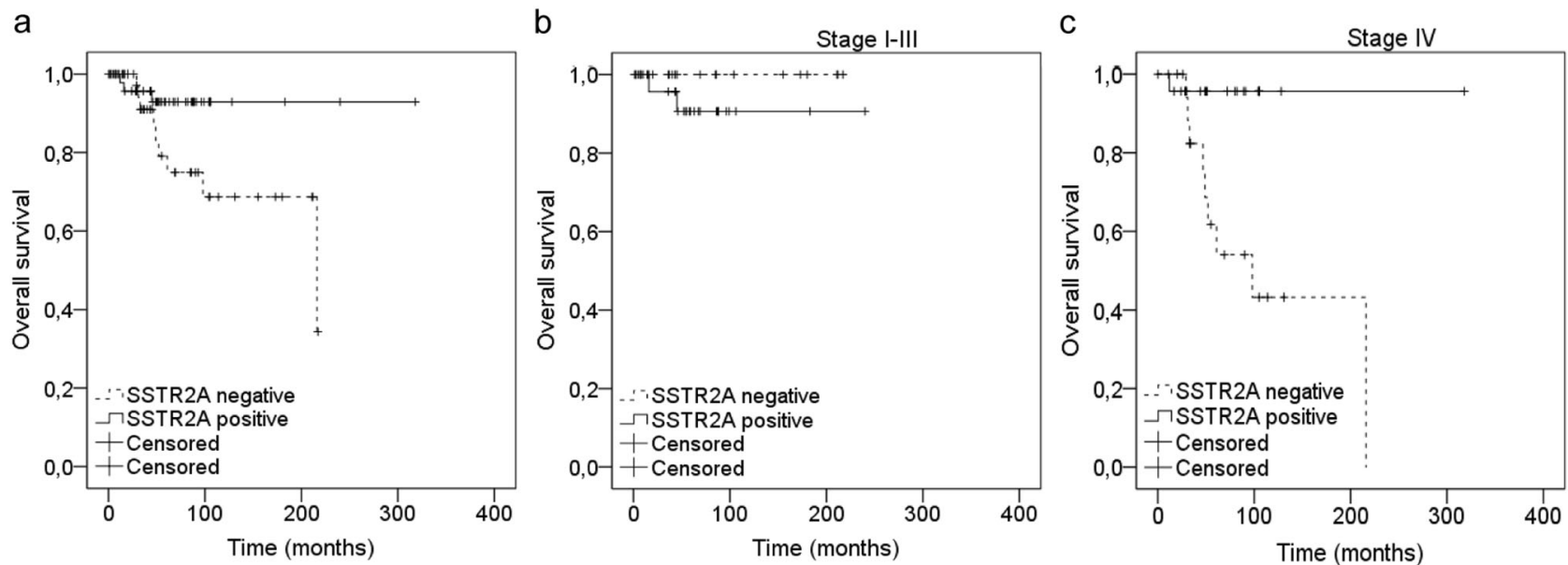

Fig. 4 Kaplan-Meier overall survival curves for patients with SSTR2A-positive and negative MTC. a Analyses over total group of 114 patients. b Analyses over subpopulation of TNM-stage I-III patients. c Analyses over subpopulation of TNM-stage IV patients

Table 3 Multivariate Cox-regression analysis on OS

\begin{tabular}{llll}
\hline & $\begin{array}{l}\text { Events/ } \\
\text { patients }\end{array}$ & $\begin{array}{l}\text { Adjusted hazard ratio (95\% } \\
\text { CI) }\end{array}$ & $p$-value \\
\hline $\begin{array}{llll}\text { SSTR2A } \\
\text { Negative }\end{array}$ & $9 / 43$ & 1 & 0.05 \\
Positive & $3 / 51$ & $0.3(0.1-1.0)$ & \\
Stage & & & 0.04 \\
I-III & $2 / 49$ & 1 & \\
IV & $10 / 45$ & $5.0(1.1-23.0)$ & \\
\hline
\end{tabular}

value. Since necrosis was only present in 11 patients (7.9\%), it is not suitable as a prognostic factor and was, therefore, not included in the multivariate Cox-regression analysis. Desmoplasia, HIF-1 $\alpha$, CAIX, Glut-1 and VEGF were not taken into account since these are all pathological variables while we wanted to see whether SSTR2A expression could add something to the existing clinical prognostic variables.

SSTR2A expression and stage significantly and independently correlated to OS, as outlined in Table 3. SSTR2A expression $\geq 1$ decreased the risk to die with a HR of 0.27 (95\% confidence interval (CI) 0.07-0.98). Having stage IV, on the other hand, increased the risk of dying with a HR of 5.05 (95\% CI 1.10-23.05).

\section{Discussion}

The aim of this study was to evaluate SSTR2A as a prognostic factor for MTC, to study distribution of SSTR2A expression within tumours and to compare expression of SSTR2A between primary tumours and corresponding lymph node metastases.
We found SSTR2A expression in 50.9\% of MTC cases. This is slightly less than the findings of Herac et al. [8] who found SSTR2A expression in $66.0 \%$ of patients. Other studies investigated SSTR2 expression in general, not SSTR2A in particular. Zatelli et al. [12] and Papotti et al. [21] studied SSTR2 expression in 51 patients and found positivity in 22 patients (43\%). Kendler et al. [22] recently found SSTR2 expression in 12 of 42 studied patients $(28.6 \%)$. Taken together, approximately half of patients seem to show SSTR2 expression.

In survival analysis, SSTR2A expression was independently associated with better OS, especially in stage IV patients. Therefore, SSTR2A expression might be a valuable prognostic marker in MTC. Interestingly, no association was found for SSTR2A expression and PFS, for which we do not have a good explanation. The more favourable prognosis accompanying SSTR2A expression could be explained by the fact that well differentiated tumours, which still express SSTRs like normal C-cells, tend to have a more indolent course of disease. Herac et al. [8] studied SSTR2A expression in 97 patients with MTC and found that SSTR2A expression could be an adverse prognostic factor since it correlated with presence of lymph node metastases. Herac et al. found that patients with lymph node metastases, were SSTR2A positive in $92.0 \%$ and negative in $8.0 \%$. In our study, patients with lymph node metastases were SSTR2A positive and negative in $57.4 \%$ and $42.6 \%$, respectively. So, in our study we also found the association between lymph node metastases and SSTR2A expression, however, contrary to Herac et al., in our study this association was not significant. The variance in our results could be explained by the fact that in the study of Herac et al. $25.8 \%$ had lymph node metastases at the time of primary surgery, whereas in our study $61.8 \%$ of patients presented with lymph node metastases [8]. 
We show for the first time that in the majority of MTC cases the distribution of SSTRs was homogeneous throughout the primary tumour. This implies that preoperatively identification of the SSTR2A status on core needle or fine needle aspiration biopsies should be possible, which has consequences for therapy strategies such as the use of PRRT. Also in practice, one can imagine that by knowing SSTR2A status of a patient who presents with stage IV disease, the treating physician might be able to give a better indication of prognosis.

Twenty-four percent of cases showed SSTR2A expression in the primary tumour, but not in $\geq 1$ of the lymph node metastases. Furthermore, $29.4 \%$ patients who did not show expression in the primary tumour, expressed SSTR2A in $\geq 1$ of the lymph node metastasis. This means that, based on our immunohistochemistry findings, SSTR2A status of the primary tumour is a poor predictor of expression in lymph node metastases, which might indicate that the SSTR2A status of the primary tumour cannot be used as a tool for clinical decision making with regard to SSTR2A nuclear imaging.

Herac et al. [8] found that desmoplasia, CAIX and HIF$1 \alpha$ were significantly correlated with SSTR2A expression. In our study, we did not find significant associations with desmoplasia and CAIX, but could confirm the association with HIF-1 $\alpha$. It is known that somatostatin and octreotide, a SSTR2A-specific SSTA, can block the activation and inhibit the effect of HIF- $1 \alpha$ and its downstream targets [23, 24]. This finding suggests a relation between the two factors, even though the correlation is not fully understood yet and this should be investigated on a cellular level.

One of the strengths of our retrospective study is the large sample size, considering MTC has a low incidence. Also, our follow-up time is long (mean 69.5 months, range 0-318 months). Long follow-up is essential since MTC is a tumour with low proliferative activity and low event rates. Furthermore, we used immunohistochemical data, which we were able to combine with clinical endpoints such as the occurrence of distant metastases and death. Limitations of our study mainly are a consequence of the retrospective design of it and the rare occurrence of MTC. In our database, we included patients from five different tertiary referral centres covering almost three decades. Therefore, in our analysis we had to use variables that were consistent over time and between centres. Since we included patients from over almost three decades we have a wide range in our follow-up period. Furthermore, guidelines for surgery have changed over the years and surgical techniques may have differed between centres, which may have resulted in not all patients getting a cervical lymph node resection. This may have caused an underestimation of lymph node-positive patients. Moreover, one might argue that whole slide analysis would be a superior technique to TMA. However, in previous studies, good concordance between TMA and whole slide analysis was found if only one punch was used, with an increasing level of concordance if more punches were considered [25]. Furthermore, for the validation of prognostic biomarkers, TMA is described as the standard [26, 27].

In conclusion, our results show that SSTR2A may be a valuable prognostic biomarker for MTC patients indicating longer OS, especially in stage IV patients. Distribution within the tumour was homogenous in the majority of cases, indicating that preoperative SSTR2A status may be assessed in core needle or fine needle aspiration biopsies. SSTR2A status of the primary MTC is a poor predictor of expression in lymph node metastases.

Acknowledgements We are grateful to Hans Morreau for his time to select the medullary thyroid carcinoma tissue of the LUMC. We would like to thank Domenico Castiliego for his contribution in the construction of the TMA.

\section{Compliance with ethical standards}

Conflict of interest The authors declare that they have no conflict of interest.

Ethical approval All procedures performed in studies involving human participants were in accordance with the ethical standards of the Institutional Review Board of the UMCU and/or national research committee and with the 1964 Helsinki declaration and its later amendments or comparable ethical standards.

Informed consent Informed consent was not obtained from individual participants included in the study. Following the national guidelines with respect to leftover tissue, informed consent was not necessary [19].

Open Access This article is distributed under the terms of the Creative Commons Attribution 4.0 International License (http://crea tivecommons.org/licenses/by/4.0/), which permits use, duplication, adaptation, distribution, and reproduction in any medium or format, as long as you give appropriate credit to the original author(s) and the source, provide a link to the Creative Commons license, and indicate if changes were made.

\section{References}

1. M. Cakir, A.B. Grossman, Medullary thyroid cancer: molecular biology and novel molecular therapies. Neuroendocrinol 90, 323-348 (2009)

2. E. Kebebew, P.H. Ituarte, A.E. Siperstein et al. Medullary thyroid carcinoma: clinical characteristics, treatment, prognostic factors, and a comparison of staging systems. Cancer 88, 1139-1148 (2000)

3. S.A. Wells Jr, S.L. Asa, H. Dralle et al. Revised American Thyroid Association guidelines for the management of medullary thyroid carcinoma. Thyroid 25, 567-610 (2015)

4. C. Scollo, E. Baudin, J.P. Travagli et al. Rationale for central and bilateral lymph node dissection in sporadic and hereditary medullary thyroid cancer. J. Clin. Endocrinol. Metab. 88, 2070-2075 (2003) 
5. M.C. Zatelli, D. Piccin, F. Tagliati et al. Selective activation of somatostatin receptor subtypes differentially modulates secretion and viability in human medullary thyroid carcinoma primary cultures: potential clinical perspectives. J. Clin. Endocrinol. Metab. 91, 2218-2224 (2006)

6. E.J. Kuo, S. Sho, N. Li et al. Risk factors associated with reoperation and disease-specific mortality in patients with medullary thyroid carcinoma. JAMA Surg. 153, 52-59 (2018)

7. L. Lodewijk, P. Van Diest, Van, P. der Groep et al. Expression of HIF-1alpha in medullary thyroid cancer identifies a subgroup with poor prognosis. Oncotarget 8, 28650-28659 (2017)

8. M. Herac, B. Niederle, M. Raderer et al. Expression of somatostatin receptor $2 \mathrm{~A}$ in medullary thyroid carcinoma is associated with lymph node metastasis. APMIS 124, 839-845 (2016)

9. N. Kimura, M. Pilichowska, F. Date et al. Immunohistochemical expression of somatostatin type $2 \mathrm{~A}$ receptor in neuroendocrine tumors. Clin. Cancer Res. 5, 3483-3487 (1999)

10. E. Mato, X. Matias-Guiu, A. Chico et al. Somatostatin and somatostatin receptor subtype gene expression in medullary thyroid carcinoma. J. Clin. Endocrinol. Metab. 83, 2417-2420 (1998)

11. M. Korner, B. Waser, A. Schonbrunn et al. Somatostatin receptor subtype 2A immunohistochemistry using a new monoclonal antibody selects tumors suitable for in vivo somatostatin receptor targeting. Am. J. Surg. Pathol. 36, 242-252 (2012)

12. M.C. Zatelli, E.C. Degli Uberti, Somatostatin receptors: from basic science to clinical approach--thyroid. Dig. Liver. Dis. 36 (Suppl 1), S86-S92 (2004)

13. C. Mahler, J. Verhelst, M. De Longueville et al. Long-term treatment of metastatic medullary thyroid carcinoma with the somatostatin analogue octreotide. Clin. Endocrinol. (Oxf.). 33, 261-269 (1990)

14. J. Strosberg, G. El-Haddad, E. Wolin et al. Phase 3 trial of (177) Lu-dotatate for midgut neuroendocrine tumors. N. Engl. J. Med. 376, 125-135 (2017)

15. F. Vaisman, P.H. Rosado de Castro, F.P. Lopes et al. Is there a role for peptide receptor radionuclide therapy in medullary thyroid cancer? Clin. Nucl. Med. 40, 123-127 (2015)

16. C. Waldherr, T. Schumacher, M. Pless et al. Radiopeptide transmitted internal irradiation of non-iodophil thyroid cancer and conventionally untreatable medullary thyroid cancer using. Nucl. Med. Commun. 22, 673-678 (2001)

17. L. Bodei, D. Handkiewicz-Junak, C. Grana et al. Receptor radionuclide therapy with 90Y-DOTATOC in patients with medullary thyroid carcinomas. Cancer Biother. Radiopharm. 19, 65-71 (2004)

18. A. Salavati, A. Puranik, H.R. Kulkarni et al. Peptide receptor radionuclide therapy (PRRT) of medullary and nonmedullary thyroid cancer using radiolabeled somatostatin analogues. Semin. Nucl. Med. 46, 215-224 (2016)

19. P.J. Van Diest, No consent should be needed for using leftover body material for scientific purposes. For. BMJ. 325, 648-651 (2002)

20. M. Volante et al. Somatostatin receptor type 2A immunohistochemistry in neuroendocrine tumors: a proposal of scoring system correlated with somatostatin receptor scintigraphy. Mod. Pathol. 20, 1172-1182 (2007)

21. M. Papotti, U. Kumar, M. Volante et al. Immunohistochemical detection of somatostatin receptor types 1-5 in medullary carcinoma of the thyroid. Clin. Endocrinol. (Oxf.). 54, 641-649 (2001)

22. D.B. Kendler, M.L. Araujo Jr., R. Alencar et al. Somatostatin receptor subtype 1 might be a predictor of better response to therapy in medullary thyroid carcinoma. Endocrine 58, 474-480 (2017)

23. K. Villaume, M. Blanc, G. Gouysse et al. VEGF secretion by neuroendocrine tumor cells is inhibited by octreotide and by inhibitors of the PI3K/AKT/mTOR pathway. Neuroendocrinology 91, 268-278 (2010)

24. S. Mei, M. Cammalleri, D. Azara et al. Mechanisms underlying somatostatin receptor 2 down-regulation of vascular endothelial growth factor expression in response to hypoxia in mouse retinal explants. J. Pathol. 226, 519-533 (2012)

25. G. Sauter, R. Simon, K. Hillan, Tissue microarrays in drug discovery. Nat. Rev. Drug. Discov. 2, 962-972 (2003)

26. S. Hassan, C. Ferrario, A. Mamo, M. Basik, Tissue microarrays: emerging standard for biomarker validation. Curr. Opin. Biotechnol. 19, 19-25 (2008)

27. D. Voduc, C. Kenney, T.O. Nielsen, Tissue microarrays in clinical oncology. Semin. Radiat. Oncol. 18, 89-97 (2008) 